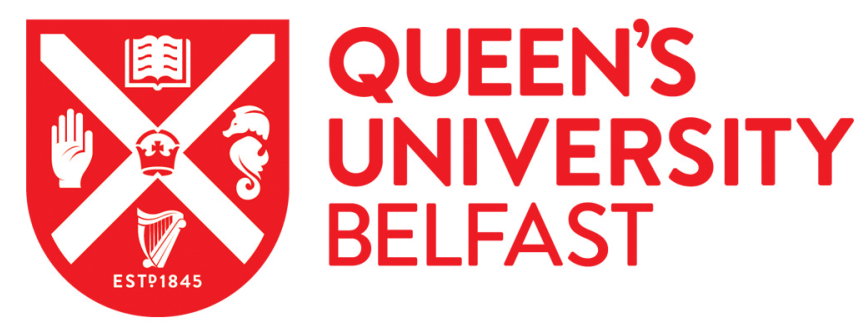

\title{
Laboratory Diagnosis and Characterization of Fungal Disease in Patients with Cystic Fibrosis (CF): A Survey of Current UK Practice in a Cohort of Clinical Microbiology Laboratories
}

Boyle, M., Moore, J. E., Whitehouse, J. L., Bilton, D., \& Downey, D. G. (2018). Laboratory Diagnosis and Characterization of Fungal Disease in Patients with Cystic Fibrosis (CF): A Survey of Current UK Practice in a Cohort of Clinical Microbiology Laboratories. Mycopathologia. https://doi.org/10.1007/s11046-018-0251-z

Published in:

Mycopathologia

Document Version:

Peer reviewed version

Queen's University Belfast - Research Portal:

Link to publication record in Queen's University Belfast Research Portal

Publisher rights

() 2018 Springer Science=Business Media B. V., part of Springer Nature.

This work is made available online in accordance with the publisher's policies. Please refer to any applicable terms of use of the publisher.

\section{General rights}

Copyright for the publications made accessible via the Queen's University Belfast Research Portal is retained by the author(s) and / or other copyright owners and it is a condition of accessing these publications that users recognise and abide by the legal requirements associated with these rights.

Take down policy

The Research Portal is Queen's institutional repository that provides access to Queen's research output. Every effort has been made to ensure that content in the Research Portal does not infringe any person's rights, or applicable UK laws. If you discover content in the

Research Portal that you believe breaches copyright or violates any law, please contact openaccess@qub.ac.uk. 


\title{
LABORATORY DIAGNOSIS AND CHARACTERIZATION OF FUNGAL DISEASE IN PATIENTS WITH CYSTIC FIBROSIS (CF):
}

\section{A SURVEY OF CURRENT UK PRACTICE IN A COHORT OF CLINICAL MICROBIOLOGY LABORATORIES}

\author{
Maeve Boyle ${ }^{\mathrm{a}}$, John E. Moore ${ }^{\mathrm{a}, \mathrm{b}, \mathrm{c}, \mathrm{d}^{*}}$, Joanna L. Whitehouse $^{\mathrm{e}}$, \\ Diana Bilton ${ }^{\mathrm{f}}$ and Damian G. Downey ${ }^{\mathrm{a}, \mathrm{d}}$
}

${ }^{a}$ Northern Ireland Regional Adult Cystic Fibrosis Centre, Level 8, Belfast City Hospital, Lisburn Road, Belfast, BT9 7AB, Northern Ireland, UK,

${ }^{\mathrm{b}}$ Northern Ireland Public Health Laboratory, Department of Bacteriology, Belfast City Hospital, Lisburn Road, Belfast, BT9 7AD, Northern Ireland, UK,

${ }^{c}$ School of Biomedical Sciences, Ulster University, Cromore Road, Coleraine, BT52 1SA, Northern Ireland, UK,

${ }^{d}$ Centre for Experimental Medicine, Queen's University, 97 Lisburn Road, Belfast BT9 7BL, Northern Ireland, UK,

e West Midlands Adult CF Centre, Heart of England NHS Foundation Trust, Bordesley Green East, Birmingham, B9 5SS, UK,

${ }^{\mathrm{f}}$ Department of Respiratory Medicine, Royal Brompton Hospital, London, UK.

Key words: cystic fibrosis; microbiology; fungal; Aspergillus; Scedosporium

\section{*corresponding author:-}

Professor John E. Moore,

Northern Ireland Public Health Laboratory,

Department of Bacteriology,

Belfast City Hospital,

Belfast, BT9 7AD,

Northern Ireland.

\begin{tabular}{|c|c|}
\hline Tel: & +44 (28) 90329241 \\
\hline Fax: & $+44(28) 90263991$ \\
\hline E-mail: & j.moore@qub.a \\
\hline
\end{tabular}


There is much uncertainty as to how fungal disease is diagnosed and characterized in patients with cystic fibrosis (CF). A 19 question anonymous electronic questionnaire was developed and distributed to ascertain current practice in clinical microbiology laboratories providing a fungal laboratory service to CF centres in the UK. Analyses of responses identified the following: (i) current UK laboratory practice, in general, follows the current guidelines, but the scope and diversity of what is currently being delivered by laboratories far exceeds what is detailed in the guidelines; (ii) there is a lack of standardization of fungal tests amongst laboratories, outside of the current guidelines; (iii) both the UK CF Trust Laboratory Standards for Processing Microbiological Samples from People with Cystic Fibrosis and the US Cumulative Techniques and Procedures in Clinical Microbiology (Cumitech) Guidelines 43 Cystic Fibrosis Microbiology, need to be updated to reflect both new methodological innovation, as well as better knowledge of fungal disease pathophysiology in CF; (iv) there is a need for clinical medicine to decide upon a stratification strategy for the provision of new fungal assays that will add value to the physician in the optimal management of CF patients; (v) there is also a need to rationale what assays should be performed at local laboratory level and those which are best served at National Mycology Reference Laboratory

69 level; and (vi) further research is required in developing laboratory assays, that will help ascertain the clinical importance of "old" fungal pathogens, as well as "emerging" fungal pathogens. 
71 Cystic fibrosis (CF) is an autosomal recessive disease caused by mutations of the cystic fibrosis

72 transmembrane conductance regulator (CFTR) gene, which is located on the long arm of human

73 chromosome 7 [1]. All disease-causing mutations in the CFTR gene prevent the chloride ion

74 channel from functioning properly, leading to a blockage of the movement of salt and water into

75 and out of cells. As a result of this blockage, cells that line the passageways of the lungs, pancreas

76 and other organs produce abnormally thick, sticky mucus which obstructs the airways and glands,

77 causing the characteristic signs and symptoms of CF. The most life-threatening complications, in

78 CF patients, are pulmonary inflammation and infection, from bacteria and fungi becoming trapped

79 in the thick, tenacious secretions in the airways, resulting in a vicious cycle of infection and

80 inflammation. Chronic lung infection is responsible for more than $90 \%$ of deaths in adults with CF

$81[2]$.

82 Although the predominant infections associated with the CF lung are associated with bacteria,

83 yeasts and filamentous fungi are frequently recovered from respiratory specimens from these

84 patients, especially with the increased use of B. cenocepacia selective agars, which support fungal

85 detection. These bacterial selective media contain high concentrations of several antibiotics,

86 enhancing growth of highly antibiotic-resistant bacterial species, including B. cenocepacia, which

87 will inadvertently support the growth of fungi. However, it is important that laboratories do not rely

88 solely on these for the isolation of fungi, but instead employ fungal selective agar to attempt the

89 isolation of these eucaryotes.

90 There is a growing awareness amongst CF physicians of the importance of fungal infections in 
91 patients with CF, largely due to : (i) the increasing incidence of fungal disease in CF patients; (ii)

92 emerging novel mechanisms of fungal pathophysiological disease, for example, Aspergillus

93 bronchitis; (iii) the limited number of effective therapies that are available or their association with

94 dose-limiting toxicities; (iv) the fact that fewer symptoms bring the infection to the attention of the

95 patient and physician early on; and (v) the difficulties to make an early diagnosis because of the

96 lack of sensitive tests for the detection of invasive fungal infections [3]. Culturing Aspergillus

97 fumigatus, per se, is not an indication for treatment but this fungus has a wide range of clinical

98 presentations and when combined with the presence of co-habiting bacterial pathogens, such as

99 Pseudomonas aeruginosa, makes it more difficult to attribute clinical significance to the presence of

100 the fungus. Reliable laboratory detection of fungi is thus the cornerstone of subsequent clinical

101 considerations.

102 Currently it is difficult to estimate the prevalence of fungal infections in patients with CF. This is

103 due mainly to a lack of monitoring of the presence of fungi in microbiological cultures of sputum,

104 in most CF registries/databases in the UK and Europe. The US Cystic Fibrosis Foundation (CFF)

105 Registry data, however, indicates an approximate doubling of prevalence of fungi being detected in

106 the sputum of patients with CF, from 1995 through 2005, rising from approximately 6\% in 1995 to

107 approximately $13 \%$ in 2005 . Such a rise as this could be the cumulative effect of the ad hoc

108 introduction of novel and improved laboratory methods, as well as greater awareness of fungi

109 disease in CF. In the absence of such data, the clinical significance of fungi is indicated mainly

110 through reports in the scientific/medical literature. To date, the majority of reports have included 
111 clinically significant fungi such as Aspergillus spp., Scedosporium species and Exophilia

112 dermatitidis [3].

113 Evolving laboratory technology and methodologies aids in the isolation, identification and

114 characterization of aetiological agents of fungal disease in CF patients. Coupled with this, various

115 laboratory guidelines exist to guide Clinical Microbiology Laboratories, in the employment of

116 suitable techniques to employ, to support laboratory workup of respiratory specimens, and to guide

117 clinicians in patients' management. It was therefore the aim of this study to examine how a cohort

118 of NHS Clinical Microbiology Service Laboratories, supporting CF Centres in the UK were

119 performing CF fungal diagnosis.

120

121 A 19 question anonymous electronic questionnaire was developed and posted on the SurveyGizmo

122 platform for completion (see online Supplementary material). This questionnaire is also available at

123 the following link: www.surveygizmo.eu/s3/90008906/Fungal-Laboratory-Questionnaire-for-

\section{Cystic-Fibrosis}

125 The questionnaire was designed in four sections, exploring NHS service laboratory aspects of: (i)

126 fungal isolation; (ii) fungal identification; (iii) fungal characterization; and (iv) promoting best

127 practice. The questionnaire was distributed amongst NHS Consultant Respiratory Physicians and

128 NHS Consultant Paediatricians involved in the clinical care of patients with CF at recognised UK

129 CF Centres. A request was made to 94 paediatric and adult CF consultants in the UK to forward the

130 questionnaire onto their Consultant Microbiologist, who supports the CF Centre, in terms of NHS 
131 Microbiology Laboratory Service provision. Questionnaires were duly completed and returned via

132 the SurveyGizmo platform for analyses.

134 Responses to the questionnaire were received from 11 publically-funded NHS Clinical

135 Microbiology laboratories in the UK, supporting either a CF Adult Service or a CF Paediatric

136 Service. Collated responses to the specific questions asked are shown in Table 1.

138 The goal of any clinical microbiology laboratory supporting the routine processing of sputum and

139 other respiratory specimens from CF patients, is to provide a robust and effective service, in a

140 timely and cost effective manner. Any assays that are performed need to add clinical value and aid

141 the physician in the clinical decision-making process. Driving forces, namely the development of

142 novel techniques of isolation and characterization of fungi and new insights into the

143 pathophysiology of fungal disease in CF patients, make the methodological techniques to be in a

144 constant state of evolution, thus requiring periodic rationalization to ensure NHS routine service

145 fungal assays are keeping pace with methodological innovation, as well as emerging knowledge on

146 disease driving what assays to optimally employ.

147 Currently, there are at least two laboratory standards in the UK and US, respectively namely the UK

148 CF Trust Laboratory Standards for Processing Microbiological Samples from People with Cystic

149 Fibrosis [4] and the US Cumulative Techniques and Procedures in Clinical Microbiology

150 (Cumitech) Guidelines 43 Cystic Fibrosis Microbiology [5]. Many countries may have their own 
151 national standards in place. A comparison of these standards is shown in Table 2. When we

152 compare the findings of this questionnaire, against these current guidelines for the laboratory

153 processing of sputum for fungi from CF patients, responses to this questionnaire were generally

154 within compliance of these guidelines. This study indicated that many laboratories are currently

155 performing several more assays than are presently listed in the laboratory guidelines, with a high

156 degree of non-standardization in assays not defined in the guidelines. Our questionnaire showed that

157 most laboratories are employing basic fungal detection media, mainly SDA with or without

158 antibiotics. Most laboratories are cautious about employing enhanced specific fungal culture media,

159 such as DRBC-benomyl, Sce-Sel+ or Scedo-Select III, to aid with the isolation of fungi from CF

160 sputum; none were using these recently described media [6].

161 Whilst this study received responses from 11 NHS Clinical Microbiology laboratories, this should

162 not be interpreted as being fully reflective of practice in all NHS laboratories. However, we believe

163 that the responses received from these 11 laboratories are a microcosm of UK laboratory practice

164 and a reasonable reflection of what UK laboratories are currently doing.

165 The biggest challenge resulting from analysis of this questionnaire is the lack of standardization of

166 methods across these laboratories. Previously, Borman and colleagues [7] investigated the

167 consequences of the lack of standardization of fungal methodologies across eight laboratories and

168 concluded that without more sophisticated molecular methods, the aetiological role of "rarer"

169 filamentous fungi in pulmonary exacerbations will remain hidden.

170 Most recently, the February 2018 issue of Mycopathologia (Volume 183; Issue 1) contains 25 
171 articles which are highly relevant to this area. In particular, the paper by Chen and colleagues [8]

172 discusses the challenges in laboratory detection of fungal pathogens in the airways of CF patients.

173 In this article, the authors highlight and discuss in detail the repertoire of available mycological

174 laboratory techniques (cultural and molecular methods) to support accurate isolation, identification

175 and characterization of fungal organisms from CF respiratory specimens and conclude that

176 guidelines for standardized processing of respiratory specimens are urgently needed. Following on

177 from this call for urgent standardization of methods, the paper by Coron and colleagues [9] takes on

178 this challenge to standardize cultural/isolation methods with the "MucoFong" program, whereby

179 sputa from $243 \mathrm{CF}$ patients from seven CF centres in France were studied over a 15 month period.

180 Six fungal culture media were compared and the study concluded that four of these media, namely

181 CHROMAgar Candida medium incubated at $37^{\circ} \mathrm{C}$, Sabouraud dextrose agar with chloramphenicol

182 and gentamicine at $25^{\circ} \mathrm{C}$, Sabouraud dextrose agar with chloramphenicol and cycloheximide at

$183 \quad 37^{\circ} \mathrm{C}$ and erythritol agar at $27^{\circ} \mathrm{C}$ should be employed to optimally recover fungal pathogens from

184 CF respiratory specimens. Initiatives such as the MucoFong program are extremely valuable as

185 they present an evidence-base for laboratories to move forward confidently in the knowledge that

186 they are providing an optimal service for the patients.

188 The current study highlights the following:

189 (i) that current UK laboratory practice, in general, follows the current guidelines, but that the scope 190 and diversity of what is currently being delivered by laboratories far exceeds what is detailed in the 
191 guidelines;

192 (ii) there is a lack of standardization of fungal tests amongst laboratories, outside of the current

193 guidelines;

194 (iii) as a result, both the UK CF Trust Laboratory Standards for Processing Microbiological

195 Samples from People with Cystic Fibrosis [4] and the US Cumulative Techniques and Procedures in

196 Clinical Microbiology (Cumitech) Guidelines 43 Cystic Fibrosis Microbiology [5], need to be

197 updated to reflect both new methodological innovations, as well as better knowledge of fungal

198 disease pathophysiology in CF;

199 (iv) there is a need for clinical medicine to decide upon a stratification strategy for the provision of

200 new fungal assays with added value to guide physicians for an optimal management of CF patients;

201 (v) there is a need to rationale what assays may be performed at local laboratory level and those

202 which are best served at National Mycology Reference Laboratory level;

203 and (vi) further research is required in developing laboratory assays, that will help ascertain the

204 clinical importance of "old" fungal pathogens, as well as "emerging" fungal pathogens.

205 Acknowledgements:

206 The authors wish to gratefully thank all the respondents who replied to this questionnaire.

207 ETHICAL STATEMENT:

208 No human or animal subjects were involved with this publication which as such did not require

209 ethical permission to be undertaken. 


\section{REFERENCES}

2121 Gilligan P. Microbiology of cystic fibrosis lung disease. In Cystic Fibrosis in Adults. J. R.

213 Yankaskas and M.R. Knowles (ed). Philadelphia: Lippincott-Raven Publishers, (1999) pp. 93$214 \quad 114$.

2152 Saiman L. Microbiology of early CF lung disease. Paediatr Respir Rev. 2004;5 Suppl A:S367$216 \quad$ S369.

2173 Nagano Y, Millar BC, Johnson E, Goldsmith CE, Elborn JS, Rendall J, Moore JE. Fungal 218 infections in patients with cystic fibrosis. Rev Med Microbiol. 2007;18:11-16.

2194 Anon. UK CF Trust Laboratory Standards for Processing Microbiological Samples from $220 \quad$ People with Cystic Fibrosis. Available at: https://www.cysticfibrosis.org.uk/ /media/documents/the-work-we-do/care/consensus-docswith-new-address/laboratory-standards.ashx?la=en [Last access on 24 July 2017].

2235 Gilligan PH, Kiska DL, Appelman MD. Cumitech 43, Cystic fibrosis microbiology. 224 Coordinating ed., Appelman M.D. ASM Press, Washington, D.C., 2006.

2256 Nagano Y, Millar BC, Goldsmith CE, Walker JM, Elborn JS, Rendall J, Moore JE. 226 Development of selective media for the isolation of yeasts and filamentous fungi from the 227 sputum of adult patients with cystic fibrosis (CF). J Cyst Fibros. 2008;7:566-572.

2287 Borman AM, Palmer MD, Delhaes L, Carrère J, Favennec L, Ranque S, Gangneux JP, Horré 229 R, Bouchara JP. Lack of standardization in the procedures for mycological examination of 230 sputum samples from CF patients: a possible cause for variations in the prevalence of 231 filamentous fungi. Med Mycol. 2010;48 Suppl 1:S88-97.

2328 Chen SC, Meyer W, Pashley $\mathrm{CH}$. Challenges in laboratory detection of fungal pathogens in the 233 airways of cystic fibrosis patients. Mycopathologia. 2018;183:89-100.

2349 Coron N, Pihet M, Fréalle E, Lemeille Y, Pinel C, Pelloux H, Gargala G, Favennec L, 235 Accoceberry I, Durand-Joly I, Dalle F, Huet F, Fanton A, Boldron A, Loeuille GA, Domblides 236 P, Coltey B, Pin I, Llerena C, Troussier F, Person C, Marguet C, Wizla N, Thumerelle C, Turck 237 D, Bui S, Fayon M, Duhamel A, Prévotat A, Wallaert B, Leroy S, Bouchara JP, Delhaes L. 
238 Toward the standardization of mycological examination of sputum samples in cystic fibrosis:

239 Results from a French multicenter prospective study. Mycopathologia. 2018;183:101-117. 


\section{FUNGAL ISOLATION}

1. Under which of the following circumstances does your lab attempt to isolate fungi from CF patients:

On all submitted specimens

Only on request from the submitting clinician

Other (e.g. high risk patients / post transplant)

2. Do you routinely use a selective medium for isolation of fungi from CF specimens?

3. Does your laboratory employ molecular methods for the detection of fungi from CF specimens? (either in-house or referral outside)

4. Does your laboratory employ other methods, (e.g. precipitin testing, galactomannan), different to $Q 2$ and $Q 3$ above?

\section{FUNGAL IDENTIFICATION}

5. On isolation of a fungus from a $\mathrm{CF}$ specimen, which of the following do you attempt to identify? in-house or via a Mycology Reference laboratory

6. What methods of fungal identification do you employ to achieve this?

7. What is your laboratory's practice for isolating, identifying and testing anti-fungal susceptibility on Candida spp and/or other yeasts?
Eleven (91.7\%) respondents answered on all submitted specimens and one respondent added 'any specimen except cough swabs'.

Ten respondents $(91 \%)$ routinely use the fungal selective medium, Sabouraud (SAB), for isolation of fungi from CF specimens. There was wide variation in the incubation time and temperature employed, including:

SAB for 5 days

$\mathrm{SAB}$ at 30 and $37^{\circ} \mathrm{C}$

SAB for 2 days at $37^{\circ} \mathrm{C}+5$ days at $30^{\circ} \mathrm{C}$

$\mathrm{SAB}+$ chloramphenicol at $30^{\circ} \mathrm{C}+37^{\circ} \mathrm{C}$ for 48 hours +5 days

$\mathrm{SAB}$ in $\mathrm{CO}_{2}$ at $37^{\circ} \mathrm{C}$ for 5 days

$\mathrm{SAB}+$ gentamicin + chloramphenicol at $37^{\circ} \mathrm{C}$ for 5 days

$\mathrm{SAB}$ at $35-37^{\circ} \mathrm{C}$ for 48 hours.

No laboratory reported using other CF selective media.

Two (18.2\%) laboratories employ molecular methods.

Eight $(72.7 \%)$ laboratories employ other methods (e.g. precipitin testing, galactomannan). Four of these would refer to another lab for testing, while one can do tests as special request, although their Aspergillus PCR and susceptibility testing are referred to the Mycology reference lab.

All of the 11 respondents attempt to identify all filamentous fungi that are recovered, and $3(27.3 \%)$ attempt for yeasts

Six $(54.5 \%)$ use in-house and $3(27.3 \%)$ refer to the Mycology Reference Laboratory. The remaining two respondents commented, 'a combination of conventional mycology in-house or reference laboratory for non-Candida' and, 'some ID in-house via conventional phenotypic methods, others sent to reference laboratory'.

Of those who had answered In-house, three use conventional mycology, one uses conventional and MALDI-TOF mass spectrometry, one uses microscopy, $18 \mathrm{~S}$ rDNA sequencing and the final comment was, 'phenotypic, then 18S rDNA sequencing if no identification or uncertain identification (then reference laboratory if confirmation required).

- Identification of black yeasts: 7/11 laboratories

- Identification of other yeasts: 2/11 (by MALDI-TOF mass spectrometry)

- Antifungal susceptibility testing :

- Routinely performed on all yeast species 0/11

- Performed only on non-albicans yeast species: 5/11 
- Susceptibility testing performed by reference laboratory: $7 / 11$.

- For in house in vitro susceptibility testing: $3 / 11$ use VITEK 2 and 1/11 uses Yeast One sensititre plates

\section{CHARACTERIZATION OF FILAMENTOUS FUNGI}

8 \& 9. Do you routinely perform antifungal susceptibility testing on any/all of the fungal isolates obtained from CF specimens.

If so,

10. Which Mycology Reference Lab is ?

Do they charge for this service?

How much per specimen?

What is their turnaround time?

11. Which antifungal agents would you like tested?

(Either in-house or via Mycology Reference Laboratory)

12. Do you perform any further fungal characterisation on the isolates? (e.g. molecular typing)

13. What is your laboratory policy on the storage/preservation of CF fungal isolates?

Would you be willing to archive your CF fungal isolates in a CF strain repository for sharing with others?
Seven $(63.6 \%)$ of nine respondents do not routinely perform antifungal susceptibility testing on any/all of the fungal isolates from CF specimens. Two (18.2\%) do, stating 'MIC tested on isolates from BAL samples' and, 'Yeasts if treatment clinically indicated'.

Six laboratories use the Bristol Reference Laboratory and one uses the Manchester Reference Laboratory. Five reported that they are charged for the service, while one comment stated that some tests are not charged. None of the respondents knew the cost per specimen. The turnaround time responses varied from 5 days to two weeks, depending on the isolate.

Ten $(90.9 \%)$ would like voriconazole tested, $9(81.8 \%)$ indicated ambisome and itraconazole, 7 (63.6\%) fluconazole and caspofungin, 5 (45.5\%) posaconazole, $4(36.4 \%)$ micafungin, $2(18.2 \%)$ anidulafungin and isavuconazole and one chose abelcet. Additional comments were: 'we test amphotericin not the formulations' and, 'it depends on what we are sending and what is available in our formulary'

None of the 11 of the respondents perform any other fungal characterization test (e.g. molecular typing) on the isolates

Two (18.2\%) laboratories reported (18.2\%) the all fungal isolates were preserved, $3(27.3 \%)$ reported that nothing was preserved and the remainder (6) left additional comments including storage of unusual isolates, storage of new isolates, keeping clinical isolates for a limited time due to storage capacity, keeping by specific request (beads for 2 years) and those that have been referred to the Reference Laboratory.

Nine $(90 \%)$ laboratories reported they would be willing to archive CF fungal isolates in a CF strain repository for sharing with others. The others responded negatively, citing very limited on-site capacity, however one responded that they would unwilling to archive on site but happy to send to a central fungal repository. 


\section{PROMOTING BEST PRACTICE}

14. Would your laboratory have the capacity to handle additional requests for fungal work-up from the CF clinical team?

Would you be willing to change your CF fungal work-up if presented with 'Best Practice Guidelines'?

19. Would you support the establishment of a National CF Mycology Reference Service / Laboratory?

(This could take the form of a "Virtual Reference Laboratory", with several specialist Mycology laboratories providing individual specific assays under a service level agreement)
Seven $(63.6 \%)$ laboratories reported they would have the capacity to handle additional requests for fungal work-up from the CF team, and $2(18.2 \%)$ stated they would not have capacity. Comments from the respondents highlighted that funding would be the main barrier to taking on additional requests.

All eleven respondents stated they would be willing to change their CF fungal work-up if presented with 'Best Practice Guidelines.'

Lack of financial resources was reported as the main issue with changing practice and one comment stated, 'CF samples are expensive and not properly resourced'.

Seven of the ten respondents said they would support this and one person would not. Comments given were, 'not sure if there is a need for this', 'possibly if supported by evidence' and 'undecided -(current Mycology Reference Lab do a good job and unsure of the need for duplication)'. 


\section{CF Trust Consensus Guidelines}

\section{US Cumitech Guidelines}

Fungal infections have become more prevalent in people with CF in recent years. Infection with Aspergillus spp. has long been recognised as a problem in CF, usually presenting as allergic bronchopulmonary aspergillosis. Recently it has been suggested that Aspergillus infection can cause respiratory exacerbations by stimulating a fungal-associated bronchitis that responds to specific antifungal therapies. Other fungi are increasingly recognised as complications of CF care e.g. Scedosporium apiospermum and Wangiella (now called Exophiala) dermatitidis.

Sabouraud medium should be used to enhance the recovery of fungi from respiratory samples of people with CF.

The addition of appropriate antibiotics reduces contamination rates with $P$. aeruginosa.

Plates should be incubated at $35-37^{\circ} \mathrm{C}$ in air and examined after overnight incubation and after at least another 24 hours. Prolonging cultures up to seven days and at different temperatures (e.g. $22^{\circ} \mathrm{C}$ ) may increase yield.

Correct identification of Aspergillus species is important as some are resistant to amphotericin, e.g. A. versicolor, A. nidulans, $A$. lentulus. Itraconazole-resistant Aspergillus fumigatus has been described and multiple triazole resistance has also been reported. If a patient has had prior treatment with azoles, susceptibility testing may therefore be warranted

In people with CF, the repeated isolation of Aspergillus spp., in spite of long-term treatment with antifungal drugs (e.g. itraconazole or voriconazole as steroid sparing treatment for ABPA), may indicate the need for referral of isolates to a reference laboratory for susceptibility testing.
Routine culture for fungi is not recommended for CF patients, although the organisms may grow on bacterial isolation media. When fungal isolation is attempted, it is recommended that antibacterial agents with antipseudomonal activity be incorporated into the medium.

Allergic bronchopulmonary Aspergillosis (ABPA) laboratory diagnosis may be aided by: Serum immunoglobulin E (IgE) concentration of $500 \mathrm{IU} / \mathrm{ml}$ and the presence of $\lg E$ antibodies to A.fumigatus and/or IgG antibodies to $A$. fumigatus.

Aspergillus spp. should be isolated on fungal media containing gentamicin at $30^{\circ} \mathrm{C}$ for 3 weeks in ambient air. Identification should adopt conventional identification methods. Susceptibility tests are not applicable.

The major fungal pathogen in CF patients is Aspergillus. It can grow on several of the selective bacterial media. Aspergillus can chronically infect or colonize CF patients. When Aspergillus is first detected in a bacterial culture, it should be identified to the species level and reported. After the initial isolation, the frequency of identification and reporting should be based on clinician expectations and needs. When fungal cultures are specifically requested for CF patients, selective fungal media are required because of the potential for bacterial over-growth, especially with $P$. aeruginosa. Selective fungal media containing gentamicin, amikacin, or ciprofloxacin should be used because of their activity against strains of $P$. aeruginosa and because media containing these antimicrobials will enhance the recovery of molds from CF respiratory specimens. Although Candida species are frequently recovered from CF respiratory specimens, there is no evidence that these organisms play a role in chronic CF lung disease. Therefore, Candida should not be reported for respiratory cultures from CF patients. 